\title{
Apple polyphenol extracts prevent aspirin-induced damage to the rat gastric mucosa
}

\author{
Giuseppe D'Argenio $^{1 *}$, Giovanna Mazzone ${ }^{1}$, Concetta Tuccillo ${ }^{2}$, Ilenia Grandone ${ }^{1}$, Antonietta G. Gravina ${ }^{2}$, \\ Giulia Graziani ${ }^{3}$, Vincenzo Fogliano ${ }^{3}$ and Marco Romano ${ }^{2 *}$ \\ ${ }^{1}$ Gastroenterologia, Dipartimento di Medicina Clinica e Sperimentale, Università Federico II, Naples, Italy \\ ${ }^{2}$ Dipartimento Medico Chirurgico di Internistica Clinica e Sperimentale-Gastroenterologia e C.I.R.A.N.A.D., \\ Seconda Università di Napoli, Naples, Italy \\ ${ }^{3}$ Dipartimento di Scienza degli Alimenti, Università Federico II, Naples, Italy
}

(Received 4 August 2007 - Revised 10 March 2008 - Accepted 17 March 2008 - First published online 16 May 2008)

Aspirin causes gastroduodenal ulcers and complications. Food bioactive compounds could exert beneficial effects in the gastrointestinal tract. We evaluated whether apple polyphenol extract (APE) reduced aspirin-induced injury to the rat gastric mucosa. Rats were treated with APE $\left(10^{-4} \mathrm{M}\right.$ catechin equivalent) before oral aspirin $(200 \mathrm{mg} / \mathrm{kg})$. Cyclo-oxygenase-2 (COX-2), transforming growth factor- $\alpha(\mathrm{TGF} \alpha)$ and heparinbinding epidermal-growth-factor-like growth factor (HB-EGF) mRNA and protein expression were assessed by RT-PCR and Western blot analysis, respectively; malondialdehyde (MDA) was determined by HPLC; gastric secretion was evaluated in pylorus-ligated rats. APE decreased acute and chronic aspirin injury both macroscopically and microscopically (approximately $50 \%$ decrease in lesion score; $P<0 \cdot 05$ ). Aspirin up-regulated mRNA and protein expression of COX-2 and HB-EGF, but not of TGF $\alpha$; APE reduced aspirin-induced mRNA and protein overexpression of COX-2 and HB-EGF; aspirin significantly increased gastric MDA and this effect was counteracted by APE pre-treatment. APE did not significantly affect gastric acid secretion. In conclusion, APE reduces aspirin-induced gastric injury independently of acid inhibition. We speculate that APE might be of therapeutic use in the prophylaxis of aspirin-related gastropathy.

Apple polyphenols: Aspirin: Gastric injury

Non-steroidal anti-inflammatory drugs (NSAID), including aspirin, are among the most commonly used medications in the world. Gastrointestinal (GI) morbidities represent the most common adverse effects associated with NSAID use, with serious GI tract complications occurring in $1-4 \%$ of NSAID users annually ${ }^{(1)}$. In particular, symptomatic or complicated ulcers are the most serious GI side effects. Low-dose aspirin (i.e. $<325 \mathrm{mg} / \mathrm{d}$ ) increases the risk for GI bleeding and hospitalisation and an increasing amount of literature suggests that even cardiovascular doses of aspirin increase GI risk two to four times ${ }^{(2)}$. Thus, it appears that no risk-free dose of aspirin exists and the attempts to coat or buffer aspirin to mitigate GI effects have not attenuated complications thus far ${ }^{(3)}$.

Because NSAID-induced gastric injury is acid-dependent (i.e. the higher the acid the greater the damage), attempts to reduce the risks of NSAID-associated GI toxicity include co-therapy with anti-secretory agents. Epidemiological and endoscopic studies have been suggested that proton-pump inhibitors (PPI) represent a reasonable choice for co-therapy. In particular, esomeprazole has recently been shown to significantly prevent ulcer bleeding in patients taking low-dose aspirin ${ }^{(4)}$.
However, the addition of PPI to therapy implies some risks, such as increased rates of pneumonia or hip fractures ${ }^{(5,6)}$. A recent study demonstrated that the use of long-term pharmacological gastric acid suppression is correlated with an increased risk of upper GI cancer, even though it is not completely clear whether the agent per se rather than the underlying treatment indication is the true risk factor for cancer ${ }^{(7)}$. Finally, the use of PPI as a strategy to reduce NSAID-related complications has low compliance and high costs.

The use of food bioactive compounds to reduce NSAID side effects represents a reliable approach well acceptable by the general public and that can be particularly effective for stomach disorders, as food bioactive compounds are present in the stomach at the same concentration as in the original foods without uncertainties related to their bioavailability and biotransformation.

We have recently demonstrated that an apple polyphenol extract (APE) obtained from the 'Annurca' variety has strong antioxidant properties and that apple antioxidants are able to significantly decrease the amount of injury to gastric epithelial cells in vitro brought about by reactive oxygen

Abbreviations: APE, apple polyphenol extract; COX-2, cyclo-oxygenase-2; GAPDH, glyceraldehyde-3-phosphate dehydrogenase; GI, gastrointestinal; HB-EGF, heparin-binding epidermal-growth-factor-like growth factor; MDA, malondialdehyde; NSAID, non-steroidal anti-inflammatory drugs; PPI, proton-pump inhibitor; TGF $\alpha$, transforming growth factor- $\alpha$; Tris, 2-amino-2-hydroxymethyl-propane-1,3-diol.

* Corresponding authors: Dr G. D’Argenio, fax + 3981 7462739, email dargenio@unina.it; Dr M. Romano, fax + 3981 5666714, email marco.romano@unina2.it 
species or by indomethacin ${ }^{(8)}$. Whether APE prevents aspirininduced gastric injury without affecting gastric acid secretion is not known. Should this be the case, APE might represent a safe, risk-free, low-cost, natural way to prevent GI adverse effects in those patients who are on long-term aspirin for cardiovascular problems.

The present study was therefore designed to evaluate whether pre-treatment with APE decreased aspirin-induced injury to the rat stomach and if any protective effect was contributed to by inhibition of gastric acid secretion. The expression of cyclooxygenase-2 (COX-2), transforming growth factor- $\alpha$ (TGF $\alpha$ ) and heparin-binding epidermal-growth-factor-like growth factor (HB-EGF), which are known to be involved in the maintenance of gastric mucosal integrity, was assessed following aspirin injury with or without pre-treatment with APE.

\section{Materials and methods}

\section{Preparation of apple extract}

Freeze-dried apple flesh (i.e. without skin) of the 'Annurca' variety was extracted with methanol $(5 \mathrm{ml} \mathrm{methanol} / \mathrm{g}$ freeze-dried apple flesh approximately corresponding to $10 \mathrm{~g}$ fresh apple flesh). Three successive extractions from the same apple sample were carried out at $4^{\circ} \mathrm{C}$ for $1 \mathrm{~h}$ in the dark. Methanol extracts were pooled and filtered (Millipore Millex-GN $0.2 \mu \mathrm{m}$; Millipre Corp., Billerica, MA, USA) before analysis by HPLC and MS. During filtration mainly polysaccharides and sugars are eliminated. This extract containing mainly polyphenols, but also some methanol-soluble sugars, peptides and other potentially bioactive compounds, was assayed for antioxidant activity and for measuring protective effects on cultured gastric cells.

Identification and quantification of phenolic compounds in apple flesh extracts

Phenolic compounds were separated by HPLC (ShimadzuCLASS M10; Shimadzu, Kyoto, Japan) configured with LC-10AD pumps, an SPD-M10A diode array detector and a Rheodyne injector. The mobile phase (flow rate $1 \mathrm{ml} / \mathrm{min}$ ) consisted of acidified water (0.01 M-phosphoric acid; solvent A) and methanol (solvent B). The separation was obtained by the following gradient: at $0 \mathrm{~min} 5 \% \mathrm{~B}$; at $10 \mathrm{~min}, 50 \% \mathrm{~B}$; at $20 \mathrm{~min}, 70 \% \mathrm{~B}$; at $25 \mathrm{~min}, 80 \% \mathrm{~B}$; at $30 \mathrm{~min}, 100 \% \mathrm{~B}$; at $35 \mathrm{~min}, 50 \% \mathrm{~B}$ and at $40 \mathrm{~min}, 5 \% \mathrm{~B}$. Phenolic compounds determined were $(+)$-catechin, chlorogenic acid, (-)-epicatechin, caffeic acid, rutin and phloridizin. The analytical separations were performed on a Luna reversed-phase $\mathrm{C}_{18}(5 \mu \mathrm{m})$ column $(25 \times 4.6 \mathrm{~mm}$ internal diameter; Phenomenex, Torrance, CA, USA). All solvents were for HPLC grade and degassed with $\mathrm{He}$ at room temperature before use. Catechin, (-)-epicatechin, phloridizin and rutin were detected at $280 \mathrm{~nm}$; chlorogenic acid and caffeic acid were detected at $325 \mathrm{~nm}$. Phenolic compounds were expressed in $\mathrm{mg} / 100 \mathrm{~g}$ apple fresh weight. Identification of phenolic compounds present in APE was performed by HPLC-electrospray ionisation-tandem MS analysis as described elsewhere ${ }^{(9)}$. The sum of the concentration of all compounds present in the extract was performed and the molecular weight of the catechin was considered to obtain the molar concentration of APE. Thus, the molar concentration of APE was computed assuming that the molecular weight of catechin characterised all compounds within APE.

\section{Induction of gastric mucosal damage}

Forty-five male Wistar rats weighing 180-220 g were randomly housed in wire-bottomed cages with free access to water and standard laboratory rat food. Animals were obtained from Harlan (Milan, Italy) and maintained under controlled temperature conditions of $22 \pm 1^{\circ} \mathrm{C}$ with a $12 \mathrm{~h}$ light-dark cycle. Rats were fasted $20 \mathrm{~h}$ before the experiments and allowed free access to water. The experiments were approved by the University's ethics committee.

Thirty animals (ten animals per study group) underwent intragastric administration of $1 \mathrm{ml} \mathrm{APE}$ at $10^{-4} \mathrm{M}$, or $1 \mathrm{ml}$ esomeprazole $(500 \mu \mathrm{g} / \mathrm{kg})$ or vehicle. After $1 \mathrm{~h}$, rats received aspirin $(200 \mathrm{mg} / \mathrm{kg})$ by oral administration and were killed $4 \mathrm{~h}$ later. Another fifteen rats (five rats per study group) received APE at $10^{-4} \mathrm{M}$, or esomeprazole, or vehicle as above but not aspirin. All animals were killed under anaesthesia, the stomachs were removed and opened along the greater curvature, rinsed in cold saline, and inspected for macroscopic damage. Then, the area of haemorrhagic lesions developed in the corpus mucosa was measured under a dissecting microscope with a square grid $(\times 10)$, summed per stomach, and used as a lesion score ${ }^{(8)}$. Samples for histology were taken. The investigator measuring the lesions (G. D.) was unaware of the treatments given to the animals.

\section{Histology}

Samples for light microscopy were excised from the gastric glandular epithelium at a region located $2 \mathrm{~mm}$ below the limiting ridge that separates the forestomach from the glandular epithelium along the greater curvature of the stomach. Thus, samples, fixed in formalin, were processed for each tissue. Haematoxylin and eosin-stained paraffin-embedded sections were examined in a blinded fashion by two investigators to quantify the extent of mucosal injury. Using a graded micrometer eyepiece, within each tissue block from each stomach the overall length of tissue section was measured and the corresponding percentage of length of mucosal surface injured was determined. The mucosa was considered injured if one or more of the following criteria were present: discontinuous surface, dilated gland, haemorrhage, or damage to superficial cells ${ }^{(8)}$.

\section{Protein extract preparations}

Frozen rat stomach samples were homogenised in RIPA lysis buffer $(0.1 \%$ SDS, $0.5 \%$ deoxycholate, $1 \%$ Nonidet, $100 \mathrm{~mm}-\mathrm{NaCl}, \quad 10 \mathrm{~mm}$-2-amino-2-hydroxymethyl-propane1,3-diol (Tris)- $\mathrm{HCl}(\mathrm{pH} 7 \cdot 4))$ containing a protease inhibitor cocktail (Sigma, St Louis, MO, USA), $0.5 \mathrm{~mm}$-dithiothreitol and $0.5 \%$ phenylmethylsulfonyl fluoride. After $30 \mathrm{~min}$ at $4^{\circ} \mathrm{C}$, tissue lysates were clarified by centrifugation at $14000 \mathrm{rpm}$ for $10 \mathrm{~min}$ at $4^{\circ} \mathrm{C}$. The cleared tissue lysates were collected and stored at $-80^{\circ} \mathrm{C}$ and the protein concentration of each sample was determined by the Bradford assay (Coomassie brilliant blue protein assay; Bio-Rad, Melville, NY, USA). 


\section{Antibodies}

The antibodies were purchased from the following sources:

(1) goat polyclonal IgG anti-COX-2 (UPSTATE 07-693), epitope corresponding to an amino acid sequence 584-589 of rat $\mathrm{COX}-2$;

(2) goat polyclonal IgG anti-HB-EGF (no. sc-1413; Santa Cruz Biotechnology Inc., Santa Cruz, CA, USA);

(3) goat polyclonal IgG anti-TGF $\alpha$ (sc-1338; Santa Cruz Biotechnology Inc., Santa Cruz, CA, USA).

The secondary antibody was anti-goat $\mathrm{IgG}(\mathrm{H}+\mathrm{L})$ (Vector BA 9500).

\section{Western blot analysis}

Total protein extracts $(50 \mu \mathrm{g})$ were boiled in Laemmli buffer (0.125 M-Tris-HCl (pH 6.8), $4 \%$ SDS, $20 \%$ glycerol, $10 \%$ 2-mercaptoethanol, $0.002 \%$ bromophenol blue) for $5 \mathrm{~min}$ before electrophoresis. The samples were subjected to SDS-PAGE (10 and $7 \%$ polyacrylamide) under reducing conditions. After electrophoresis, proteins were transferred to nitrocellulose membrane (Pure Nitrocellulose Membrane, 0.45 $\mu \mathrm{m}$, Bio-Rad Laboratories no. 162-0115; Bio-Rad Laboratories, Inc., Hercules, CA, USA); complete transfer was assessed using pre-stained protein standards (See Blu Plus 2 Prestained standard; Invitrogen no. LC5925; Invitrogen, Carlsbad, CA, USA). To block non-specific binding sites the membranes were treated for $1 \mathrm{~h}$ with blocking solution of $20 \%$ FBS in TNT (10 mM-Tris (pH 8), $150 \mathrm{mM}-\mathrm{NaCl}$ and $0.05 \%$ Tween-20), and then were incubated overnight at $4^{\circ} \mathrm{C}$ with the primary antibody (1) against COX-2 (diluted 1:500), (2) against HB-EGF (diluted 1:200) in $20 \%$ FBS in TNT (0.5\% Tween-20), (3) against TGF $\alpha$ (diluted 1:200) in $20 \%$ FBS in TNT $(0.5 \%$ Tween-20). The membranes were washed three times in Tween-Tris-buffered saline (TTBS; $20 \mathrm{~mm}$-Tris- $\mathrm{HCl}$ (pH 7.6), $150 \mathrm{~mm}-\mathrm{NaCl}, 0.5 \%$ Tween-20) for $30 \mathrm{~min}$. After washing with TBS, membranes were incubated for $2 \mathrm{~h}$ (at room temperature) with the biotinylated secondary antibody (anti-goat $\mathrm{IgG}$ ) (diluted 1:2000) and immunoreactive proteins were detected by development with the ABC Vectastain kit (Vector Laboratories, Burlingame, CA, USA) according to the manufacturer's instructions.

\section{RNA extraction}

Total RNA was isolated from gastric mucosa using an RNA extraction reagent, TRIzol (Invitrogen Life Technologies, Grand Island, NY, USA) according to the standard acidguanidium-phenol-chloroform method ${ }^{(10)}$.

\section{Reverse transcriptase-PCR analysis}

RT-PCR analysis was performed on total RNA isolated from gastric mucosa as described previously ${ }^{(11,12)}$. Firststrand complementary DNA was prepared using 200 units of RT (Supertranscript RT; Life Technologies, Inc., Gaithersburg, MD, USA), 1 (g of total RNA as template, and $10 \mathrm{pmol} / \mathrm{l}$ of random hexamers in the presence of $0.1 \mathrm{mM}-$ dithiothreitol, $0.5 \mathrm{~mm}$-deoxynucleotide triphosphate-lithium salt (Pharmacia, Milan, Italy), and 20 units RNase inhibitor
(Promega, Madison, WI, USA). The reaction profile was $37^{\circ} \mathrm{C}$ for $10 \mathrm{~min}$, followed by $42^{\circ} \mathrm{C}$ for $60 \mathrm{~min}$. To control for contamination by genomic DNA, all RNA samples were run in duplicate with or without the addition of RT.

RT-PCR co-amplification of rat COX-2/glyceraldehyde-3phosphate dehydrogenase (GAPDH), rat HB-EGF/GAPDH and rat $\mathrm{TGF} \alpha / \mathrm{GAPDH}$ transcripts was performed using rat COX-2 (sense, ATCGAAGAAGACTACGTGCAACA; antisense, GAGTTGAAAGCCCTCTACCA), rat HB-EGF (sense, CTCTTTCTGGCCGCAGTGTT; antisense, GCCCATGACACCTCTGTCCA), rat TGF $\alpha$ (sense, GCTAGCGCTGGGTATCCT; antisense, ACCACTCACAGTGCTTGCGG) and rat GAPDH (sense, CACAGTCAAGGCTGAGAATG; antisense, CTCAAGATTGTCAGCAATGC)-specific primers provided by PRIMM s.r.l. (Milan, Italy), and used at the final concentration of $2 \mathrm{mmol} / \mathrm{l}$. Primers were placed on different exons. PCR amplifications were performed using $50 \mathrm{ng}$ cDNA in the presence of $0.2 \mathrm{~mm}$-deoxynucleotide triphosphate (Boehringer Mannheim, Mannheim, Germany) and $0.3 \mu \mathrm{l}$ AmpliTaq DNA polymerase (Perkin Elmer, Branchburg, NJ, USA). $\mathrm{MgCl}_{2}$ was added at the final concentration of $1.5 \mathrm{mmol} / \mathrm{l}$. After an initial denaturation step $\left(95^{\circ} \mathrm{C}\right.$ for $5 \mathrm{~min}$ ), the PCR amplification was performed using the following profiles: for rat $\mathrm{COX}-2 / \mathrm{GAPDH}, 94^{\circ} \mathrm{C}$ for $1 \mathrm{~min}$, $57^{\circ} \mathrm{C}$ for $1 \mathrm{~min}$ and $72^{\circ} \mathrm{C}$ for $1 \mathrm{~min}$ for thirty-five cycles; for rat $\mathrm{HB}-\mathrm{EGF} / \mathrm{GAPDH}, 94^{\circ} \mathrm{C}$ for $1 \mathrm{~min}, 60^{\circ} \mathrm{C}$ for $1 \mathrm{~min}$ and $72^{\circ} \mathrm{C}$ for $1 \mathrm{~min}$ for thirty-five cycles; for rat TGF $\alpha / \mathrm{GAPDH}$, $94^{\circ} \mathrm{C}$ for $1 \mathrm{~min}, 58^{\circ} \mathrm{C}$ for $1 \mathrm{~min}$ and $72^{\circ} \mathrm{C}$ for $1 \mathrm{~min}$ for thirty-five cycles. GAPDH primers were added after five cycles for rat $\mathrm{HB}-\mathrm{EGF}$, and after seven cycles for rat $\mathrm{COX}-2$ and rat TGF $\alpha$. In all cases, the final extension was at $72^{\circ} \mathrm{C}$ for 10 min. PCR products were separated using $1.8 \%$ agarose gel electrophoresis and visualised by ethidium bromide staining. Sizes of the amplified fragments were estimated from migration of the $1 \mathrm{~kb}$ ladder molecular-weight marker (Life Technologies, Inc., Gaithersburg, MD, USA), and identity was assessed by restriction-enzyme digestion. To test for contamination by genomic DNA, samples were run in duplicate with $(+\mathrm{RT})$ or without $(-\mathrm{RT})$ the addition of RT.

\section{Determination of lipid peroxidation}

Lipid peroxidation was determined by measuring cellular malondialdehyde (MDA). Determination of MDA was performed by HPLC with UV detection, according to Mateos et al. ${ }^{(13)}$ with a few modifications. Briefly, stomach homogenates were prepared by mixing $0.32 \mathrm{~g}$ stomach with $1.6 \mathrm{ml}$ of $50 \mathrm{~mm}$-Tris $-\mathrm{HCl}(\mathrm{pH} \mathrm{7.4)}$ and homogenised in a mechanically driven Teflon glass homogeniser. The homogenate was sonicated for $30 \mathrm{~min}$ and centrifuged at $5000 \mathrm{~g}$ in an automatic high-speed cold centrifuge for $10 \mathrm{~min}$ at $4^{\circ} \mathrm{C}$ (Biofuge Primo R; Osterode, Heraeus, Germany). The supernatant fraction was collected and kept for estimation of MDA. A sample of $125 \mu \mathrm{l}$ supernatant fraction was placed in a $1.5 \mathrm{ml}$ Eppendorf tube and $25 \mathrm{ml} 6 \mathrm{M}-\mathrm{NaOH}$ were added. Alkaline hydrolysis of protein-bound MDA was achieved by incubating this mixture in a $60^{\circ} \mathrm{C}$ water-bath for $30 \mathrm{~min}$. Then, protein was precipitated with $62.5 \mathrm{ml}$ of $35 \%(\mathrm{v} / \mathrm{v})$ perchloric acid, and the mixture was centrifuged at $2800 \mathrm{~g}$ for $10 \mathrm{~min}$. This step was applied before the derivatisation with 2-4 dinitrophenylhydrazine to minimise sample oxidation during analysis. 
A $125 \mu \mathrm{l}$ volume of supernatant fraction was transferred to an Eppendorf vial and mixed with $12.5 \mathrm{ml} \mathrm{2-4}$ dinitrophenylhydrazine prepared as a $5 \mathrm{~mm}$ solution in $2 \mathrm{M}$-hydrochloric acid. Finally, this reaction mixture was incubated for $30 \mathrm{~min}$ at room temperature protected from light. A sample of $50 \mu \mathrm{l}$ of this reaction mixture was injected onto an Shimadzu SPD-M10A VP HPLC-DAD with a SphereClone $5 \mathrm{~mm}$ ODS $250 \times 4.6 \mathrm{~mm}$ (Phenomenex). Samples were isocratically eluted with a mixture of $0.2 \%(\mathrm{v} / \mathrm{v})$ acetic acid in deionised water, and acetonitrile $(62: 38, \mathrm{v} / \mathrm{v})$ at a flow rate of $0.8 \mathrm{ml} /$ $\mathrm{min}$ at room temperature. Chromatograms were acquired at $310 \mathrm{~nm}$. Quantification of MDA was obtained from a calibration curve constructed by injecting increasing amounts of standard MDA obtained by acid hydrolysis of tetra-ethoxypropane as described by Mateos et al. ${ }^{(14)}$. In particular, different concentrations of MDA were treated as the experimental samples (i.e. treatment with $6 \mathrm{M}-\mathrm{NaOH}$ for $30 \mathrm{~min}$ at $60^{\circ} \mathrm{C}$ followed by protein precipitation with $35 \%$ perchloric acid) before derivatisation with 2-4 dinitrophenylhydrazine for minimising possible interferences during extraction. MDA concentration was expressed as nmol MDA/g tissue.

\section{Stimulation of gastric acid secretion in rats}

Fifteen male Wistar rats weighing 180-220 g were randomly housed in wire-bottomed cages with free access to water and standard laboratory rat food. Gastric acid secretion was stimulated by pylorus ligation ${ }^{(15)}$. Under light ether anaesthesia, lasting not longer than $10 \mathrm{~min}$, the abdomen of the animals fasted for $24 \mathrm{~h}$ was opened, the stomach exposed and the pylorus ligated. In order to maintain adequate blood supply to the stomach, care was taken in order to not ligate local major blood vessels. After closing the abdomen with sterile catgut, rats were divided into three groups (five rats each) receiving by oral administration either $1.5 \mathrm{ml}$ APE at $0.1 \mathrm{M}$, or $1.5 \mathrm{ml}$ esomeprazole $(500 \mu \mathrm{g} / \mathrm{kg})$, a PPI whose mechanism of action consists in gastric acid inhibition, or an equal volume of vehicle alone. Rats were killed $1 \mathrm{~h}$ later, the stomach was removed and opened along the greater curvature, and the gastric juice collected. Gastric acid secretion was then measured as described below.

\section{Measurement of gastric acid secretion in rats}

Gastric samples were centrifuged for $10 \mathrm{~min}$ at $1500 \mathrm{rpm}$ and the volume and the $\mathrm{pH}$ of the supernatant fraction were measured. A sample of the samples was titrated with $0 \cdot 1 \mathrm{M}$ $\mathrm{NaOH}$ to $\mathrm{pH} 7$ and the gastric acid output was calculated and expressed as milliequivalents per $\mathrm{h}(\mathrm{mEq} / \mathrm{h})$.

\section{Influence of apple polyphenol extract on aspirin} bioavailability

To assess the influence of APE on aspirin bioavailability, we designed this experiment involving two groups of five male Wistar rats each treated with either aspirin at $100 \mathrm{mg} / \mathrm{kg}$ alone or with aspirin at $100 \mathrm{mg} / \mathrm{kg}$ followed by APE at $10^{-4} \mathrm{M}$. After $2 \mathrm{~h}$, plasma samples were analysed for their content of aspirin using a validated HPLC method with a limit of quantification of $10 \mathrm{ng} / \mathrm{ml}$. Inter-assay CV was between 1.3 and $3.3 \%$. Then $500 \mu \mathrm{l}$ plasma, $100 \mu \mathrm{l}$ internal standard (aspirin, $10 \mu \mathrm{g} / \mathrm{ml}$ ) and $10 \mu \mathrm{l}$ propionic anhydride were mixed and incubated at $37^{\circ} \mathrm{C}$ for $60 \mathrm{~min}$. After addition of $1.0 \mathrm{ml}$ hydrochloric acid $(0.1 \mathrm{~mol} / \mathrm{l})$, the samples were extracted with $6 \mathrm{ml}$ diethyl ether for $25 \mathrm{~min}$. After centrifugation $\left(10 \mathrm{~min} ; 2500 \mathrm{~g} ; 10^{\circ} \mathrm{C}\right)$ the organic phase was transferred into a new glass tube and evaporated to dryness under a gentle stream of $\mathrm{N}_{2}$ at $55^{\circ} \mathrm{C}$. The residue was dissolved in $1.25 \mathrm{ml}$ of mobile phase and $17 \mu \mathrm{l}$ were injected into the HPLC system under the following HPLC conditions: temperature, ambient; mobile phase, $5.6 \mathrm{~g}$ triethylamine, $32 \mathrm{~g}$ acetic acid (96\%), $208 \mathrm{~g}$ acetonitrile, $3600 \mathrm{~g}$ HPLC water; flow, $0.3 \mathrm{ml} / \mathrm{min}$; wavelength, excitation $315 \mathrm{~nm}$, emission $430 \mathrm{~nm}$.

\section{Statistical analysis}

Data are expressed as mean values and standard deviations. Significance of differences was assessed by the Student's $t$ test for comparison between two means. Differences were considered to be significantly different if $P<0 \cdot 05$.

\section{Results}

Effect of apple polyphenol extract or esomeprazole on aspirin-induced injury in rats

Aspirin ( $200 \mathrm{mg} / \mathrm{kg}$ by mouth) induced the appearance of multiple visible gastric erosions, ranging $2-10 \mathrm{~mm}$ in length and about $1 \mathrm{~mm}$ in width (Fig. 1 (a)). APE at $10^{-4} \mathrm{M}$ given $1 \mathrm{~h}$ before aspirin prevented macroscopic injury to the rat glandular stomach (Fig. 1 (b)). Quantitatively, APE pre-treatment caused an approximate $45 \%$ decrease in the extent of macroscopic injury compared with control untreated rats $(P<0.05$; Fig. 2 (a)). Also, APE pre-treatment partially prevented microscopic injury brought about by aspirin decreasing the extent of damage to the superficial epithelium and to the glandular stomach by approximately $40 \%(P<0.05$; Fig. 2 (b)). Comparable protective effects were observed following pre-treatment with esomeprazole (Fig. 2).

We also asked whether APE was able to prevent injury brought about by chronic administration of aspirin. Therefore, ten more animals (five per each study group) were given aspirin at $100 \mathrm{mg} / \mathrm{kg}$ per $\mathrm{d}$ by oral administration for $7 \mathrm{~d}$ and concomitantly treated with APE at $10^{-4} \mathrm{M}$ or vehicle (ethanol $3 \%$ ) as control, administered in drinking water. Animals were then killed $4 \mathrm{~h}$ after the last dosing of aspirin. We found that APE exerted a protective effect, both at the macroscopic and histological level, comparable with that observed following acute aspirin administration (data not shown), and therefore the subsequent experiments were only performed in the acute model of injury.

\section{Effect of apple polyphenol extract and aspirin on cyclo-oxygenase-2, transforming growth factor- $\alpha$ and heparin-binding epidermal-growth-factor-like growth factor expression}

Epidermal growth factor-related peptides, including TGF $\alpha$ and HB-EGF, are known to play a major role in the maintenance of GI mucosa integrity, by preventing exogenous injury to the stomach and by accelerating gastric mucosal repair ${ }^{(16,17)}$. Also, COX-2-generated PG are relevant as to the recovery 
(a)

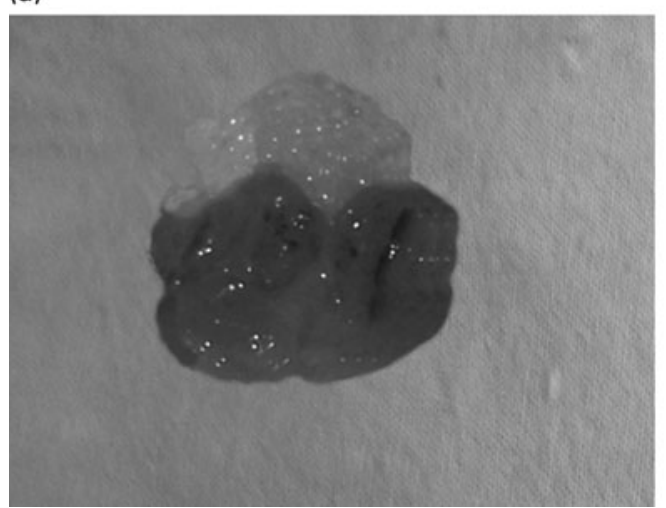

(b)

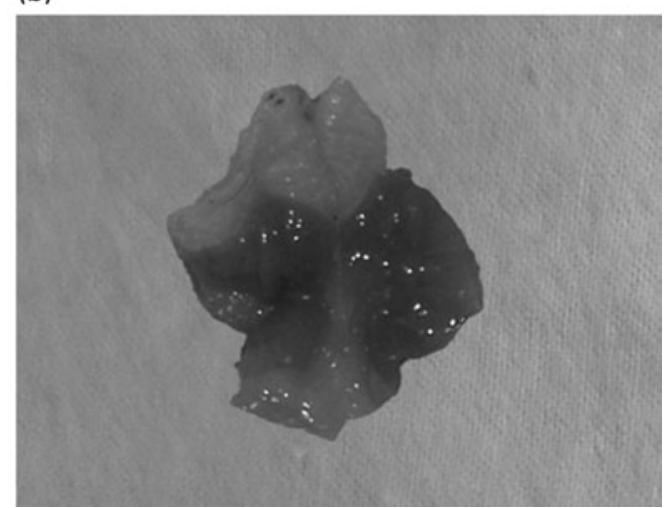

Fig. 1. Apple polyphenol extracts (APE) prevented aspirin injury to the rat gastric mucosa. Rats were pre-treated with $A P E$ at $10^{-4} \mathrm{M}$ or vehicle (control) and then with aspirin at $200 \mathrm{mg} / \mathrm{kg}$ body weight orally and killed $4 \mathrm{~h}$ later. (a) Macroscopic appearance of gastric mucosa following aspirin treatment in control vehicle-treated rats. (b) Macroscopic appearance of the gastric mucosa following aspirin treatment in APE-treated rats.

of gastric mucosal normal architecture following injury ${ }^{(18)}$. We therefore studied whether APE, in a concentration that exerted protective effects, was able to affect COX-2, $\mathrm{TGF} \alpha$ and HB-EGF expression in the gastric mucosa. APE at $10^{-4} \mathrm{M}$ did not affect COX-2, HB-EGF or TGF $\alpha$ mRNA expression (Fig. 3). Orally delivered aspirin up-regulated COX-2 (Fig. 3 (a)) and HB-EGF (Fig. 3 (b)), but not TGF $\alpha$ mRNA expression. Pre-treatment with APE at $10^{-4} \mathrm{M}$ prevented aspirin-induced up-regulation of $\mathrm{COX}-2$ and HB-EGF mRNA levels (Fig. 3 (a) and (b), respectively). Similarly, aspirin up-regulated protein expression of COX-2 and HB-EGF and this effect was prevented by APE pretreatment (Fig. 4).

\section{Effect of apple polyphenol extract on aspirin-induced lipid peroxidation}

We have previously shown that APE prevented lipid peroxidation as assessed by determination of MDA in gastric mucosal cells exposed to reactive oxygen species, in vitro ${ }^{(8)}$. Therefore, we assessed whether aspirin affected MDA gastric levels
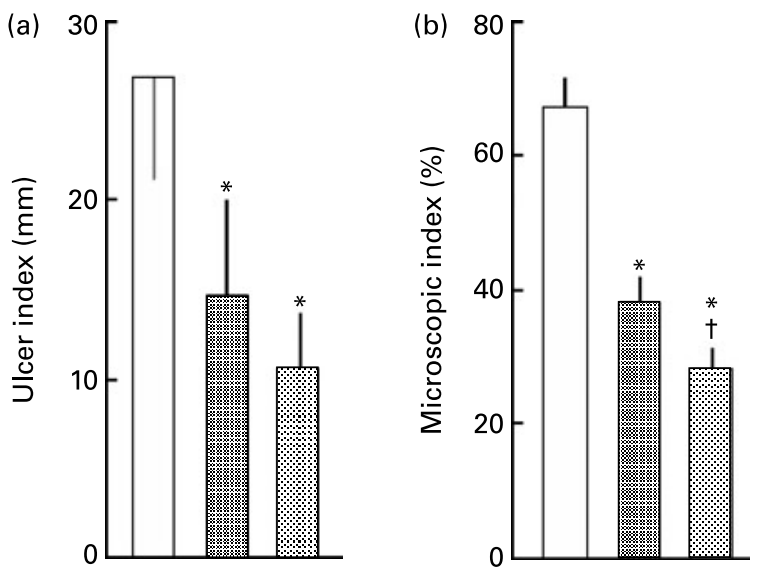

Fig. 2. Quantification of macroscopic (a) and microscopic (b) aspirin-induced injury in control vehicle- $(\square)$, esomeprazole- (圈) and apple polyphenol extract (APE; ) -treated rats. Values are means for ten rats per group, with standard deviations represented by vertical bars. * Mean value was significantly different from that of the control group $(P<0.05)$. †Mean value was significantly different from that of the APE-treated rats $(P<0.05)$. and whether APE was able to counteract any aspirin-induced change in MDA levels. Fig. 5 shows that a basal amount of MDA was formed independently of the oxidative stress induced by aspirin. Nevertheless aspirin-induced injury caused a significant, two-fold increase in MDA gastric levels. Also, APE pre-treatment significantly counteracts aspirininduced lipid peroxidation (Fig. 5).

Effect of apple polyphenol extract or esomeprazole on pylorus ligation-stimulated gastric secretion

Aspirin-induced gastric mucosal damage is acid-dependent ${ }^{(19)}$, i.e. the higher the acidity, the greater the damage. We therefore determined whether a protective concentration of APE affected gastric secretion. Fig. 6 shows that the gastric acid output determined at $1 \mathrm{~h}$ following pylorus ligation in rats was not significantly affected by APE at $10^{-4} \mathrm{M}$. In contrast, esomeprazole almost completely suppressed gastric acid secretion (Fig. 6).

\section{Influence of apple polyphenol extract on aspirin bioavailability}

Aspirin's beneficial effects (i.e. anti-inflammatory, analgesic and anti-platelet effects) depend on systemic delivery of the drug following absorption through the GI tract. We therefore asked whether APE, while decreasing aspirin injury to the stomach, might interfere with aspirin bioavailability, thus potentially decreasing its pharmacological effects. Aspirin plasma levels at $2 \mathrm{~h}$ following oral administration of aspirin at $100 \mathrm{mg} / \mathrm{kg}$ were $234(\mathrm{SD} 77) \mathrm{ng} / \mathrm{ml}$ for the aspirin plus vehicle group (i.e. five animals) and 262 (SD 38) ng/ml for the aspirin plus APE group (i.e. five animals), respectively. This difference was not statistically significant.

\section{Discussion}

We have shown that a food extract mainly consisting of a mixture of polyphenols obtained from the 'Annurca' apple significantly prevents aspirin-induced damage to the rat gastric mucosa. We also showed that this protective effect is independent of inhibition of gastric acid secretion and seems at least in part to be contributed to by the antioxidant activity of APE. 
(a)

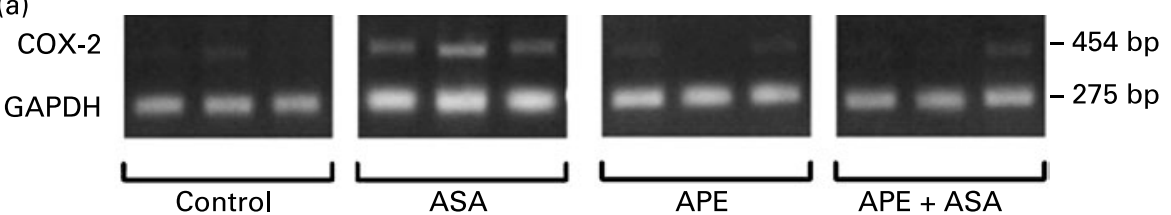

(b)
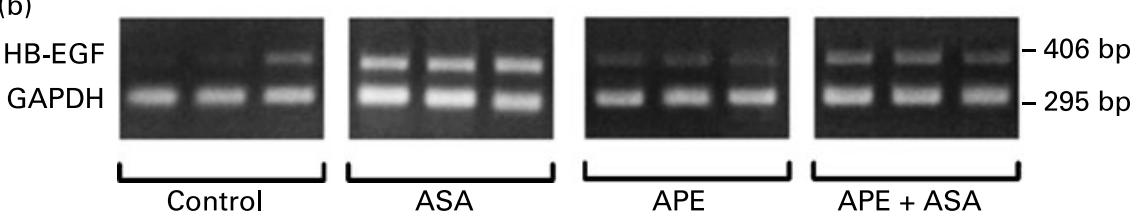

Fig. 3. Effects of aspirin (ASA) and apple polyphenol extracts (APE) on cyclo-oxygenase-2 (COX-2) and heparin-binding epidermal-growth-factor-like growth factor (HB-EGF) expression in the rat gastric mucosa. Representative RT-PCR co-amplification of COX-2/glyceraldehyde-3-phosphate dehydrogenase (GAPDH) (a) or HB-EGF/GAPDH (b) transcripts in the gastric mucosa of control untreated rats, and ASA-, APE- or APE + ASA-treated rats.

The use of NSAID, including low-dose aspirin, continues to be associated with an unacceptable risk for GI ulceration and bleeding. The most effective strategy to reduce NSAIDassociated gastric toxicity consists of the use of anti-secretory agents, including both H2-receptor antagonists or PPI. In particular, the use of NSAID combined with PPI has been adopted widely in clinical practice and it is estimated that PPI use could reduce the rate of endoscopic NSAID-related ulcers by $90 \%{ }^{(20)}$. However, prolonged use of PPI is costly and might carry some risk ${ }^{(5-7)}$. The present study shows that a natural product obtained from a variety of apple typical of Southern Italy (i.e. the 'Annurca' apple) significantly decreased the extent of gross and histological damage brought about by acute or chronic aspirin to the rat gastric mucosa, thus indicating that there might be a low-cost, effective and safe means of addressing the issue of the prevention of NSAID gastropathy. This observation is particularly relevant on clinical grounds, considering that aspirin is among the most used NSAID worldwide with a daily defined dose of 41.85 per 1000 individuals per d compared with 0.15 per 1000 people per $d$ for indomethacin, an NSAID whose mechanism of gastric injury is different in part compared with that underlying aspirin-induced damage ${ }^{(21)}$. The clinical relevance of our observation is further strengthened by the fact that APE-induced gastric protection is independent of inhibition of gastric acid secretion. In fact, APE, at a gastro-protective concentration, was not able to inhibit gastric acid secretion induced by pylorus ligation in the rat. Conversely, PPI or H2receptor blockers cannot be considered true gastro-protective agents because their protective effect against NSAID injury strictly depends on their acid-inhibitory ability. In support of this concept, in the present study, esomeprazole, a powerful inhibitor of acid secretion, which is used to prevent aspirininduced gastric injury ${ }^{(4)}$, exerted a protective effect comparable with that obtained with APE but this was at the expenses of a profound, almost complete, inhibition of gastric acid secretion. Sustained and prolonged acid inhibition may increase the susceptibility of GI tract to infections and may increase the risk of upper GI cancer ${ }^{(7)}$.

Dietary antioxidants have previously been shown to prevent NSAID-induced injury to the rat stomach. In particular Singh et al. reported that $\beta$-carotene was protective against indomethacin-induced injury in the rat ${ }^{(22)}$. Alarcon de la Lastra et al. showed that extra-virgin olive oil-enriched diets prevented indomethacin-induced gastric damage in rats ${ }^{(23)}$. Furthermore, the synthetic flavonoid meciadanol has been shown to prevent ethanol- and aspirin-induced gastric injury in the rat $^{(24)}$. We also demonstrated that apple polyphenols prevented gastric damage induced by reactive oxygen species or by parenteral indomethacin and that this effect was mainly

(a)

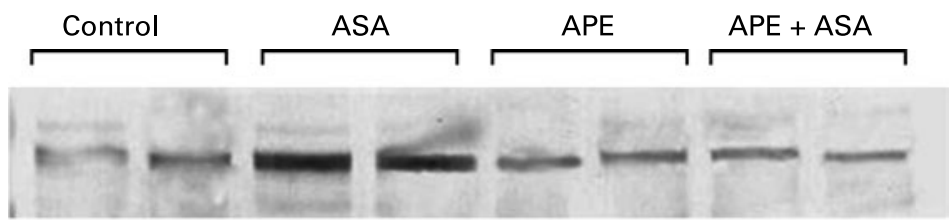

$72 \mathrm{kDa}$

(b)

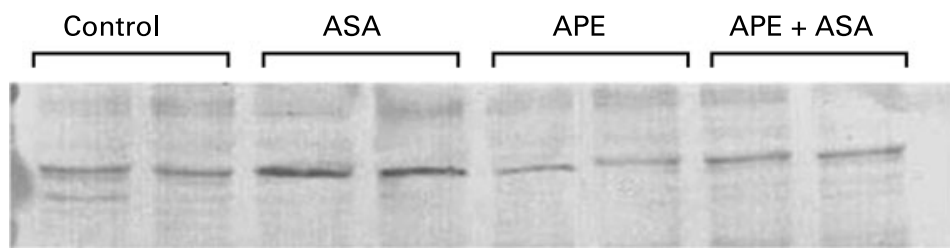

HB-EGF

$21 \mathrm{kDa}$

Fig. 4. Effects of aspirin (ASA) and apple polyphenol extracts (APE) on (a) cyclo-oxygenase-2 (COX-2) and (b) heparin-binding epidermal-growth-factor-like growth factor (HB-EGF) protein expression in the rat gastric mucosa. Representative immuno-blots from gastric mucosa were obtained from control untreated rats, and ASA-, APE- or APE + ASA-treated rats. 


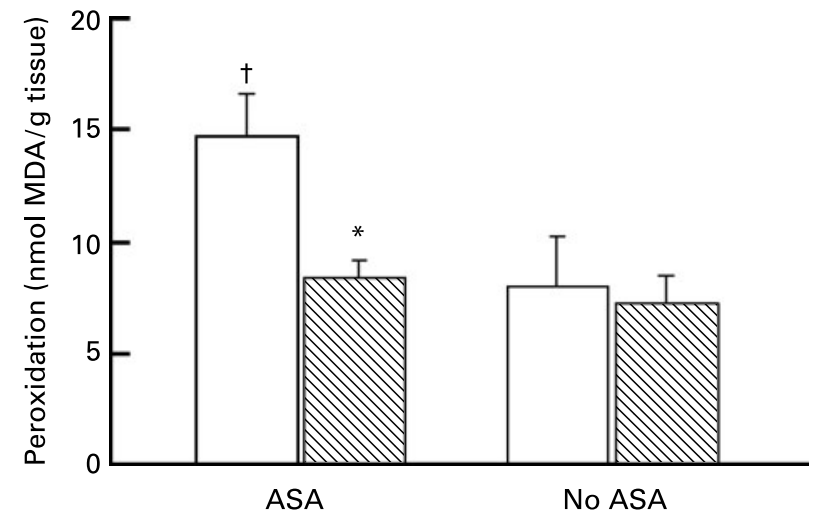

Fig. 5. Effects of apple polyphenol extracts (APE; $\triangle$ ) on lipid peroxidation compared with control treatment $(\square)$ in rat gastric mucosa. APE prevented aspirin (ASA)-induced increase in gastric malondialdehyde (MDA), a marker of lipid peroxidation. Values are means, with standard deviations represented by vertical bars. ${ }^{*}$ Mean value was significantly different from that of the control group $(P<0.05)$. † Mean value was significantly different from that of the group that received no ASA $(P<0.05)$

due to catechin, epicatechin and cholorogenic acid ${ }^{(8)}$. The present study gives further support to the notion that dietary antioxidants might be of use in the clinical setting and shows that apple polyphenols are capable of preventing gastric injury brought about by an agent (i.e. aspirin) whose damaging effect is mainly due to its topical irritative action, rather than being a result of PG deficiency, without affecting gastric acid secretion $^{(25)}$.

The mechanism whereby APE prevents aspirin injury to the gastric mucosa is still not completely clear. We previously showed that APE prevented oxidative damage to cultured gastric epithelial cells ${ }^{(8)}$. This protective effect was associated with an increase in the intracellular antioxidant activity together with a decrease in the lipid peroxidation induced by reactive oxygen species ${ }^{(8)}$. The present study suggests that the antioxidant property of the major APE phenolic constituents (i.e. chlorogenic acid, catechin and epicatechins) may play a role in the protection against aspirin injury. In fact, APE significantly counteracted the aspirin-induced increase in the gastric concentration of MDA, a marker of lipid peroxidation. This hypothesis is further supported by studies showing that the anti-ulcerogenic effects of dietary antioxidants are at least in part due to their ability to inhibit lipid peroxidation, to scavenge reactive oxygen species and/or to modulate leucocyte function ${ }^{(26)}$. Also, phenolic compounds are known to act as reactive oxygen species scavengers, hydrogen-donating compounds, single oxygen quenchers and metal ion chelators ${ }^{(27)}$, and these effects may all contribute to their antioxidant effects. APE might exert its protective effect by increasing total glutathione content in the gastric epithelium $^{(23)}$ and also by scavenging the NO radicals which are abundantly produced in the stomach at low $\mathrm{pH}^{(28)}$. However, in this respect it is worthy of note that no nitrate derivatives of chlorogenic acid were found in the stomachs of APE-treated rats (data not shown).

Because aspirin's beneficial effects (i.e. anti-inflammatory, analgesic and anti-platelet effects) depend on systemic delivery of the drug following absorption through the GI tract, we also explored the possibility that APE, while decreasing aspirin injury to the stomach, might interfere with aspirin

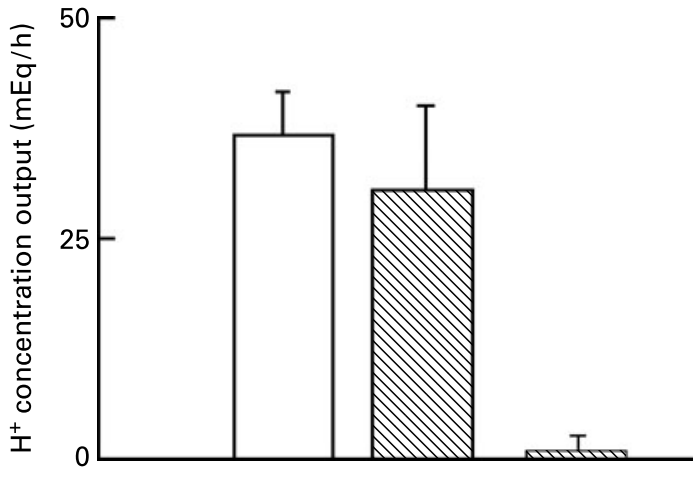

Fig. 6. Effect of apple polyphenol extracts (APE) on gastric acid secretion. Gastric acid secretion was measured in pylorus-ligated rats $1 \mathrm{~h}$ after oral administration of APE at $10^{-4} \mathrm{M}(\varangle)$, esomeprazole (ब) or vehicle (control; $\square$ ). Gastric acid secretion is expressed as acid output (i.e. $\mathrm{mEq} / \mathrm{h}$ ). Values are means, with standard deviations represented by vertical bars.

bioavailability, thus potentially decreasing its effectiveness. However, the present study indicates that APE does not affect blood levels of aspirin following oral administration.

Epidermal growth factor receptor ligands as well as COX-2 products are involved in the maintenance of gastric mucosal integrity, by preventing exogenous injury and by promoting gastric mucosal healing ${ }^{(16-18)}$. We therefore sought to investigate whether APE treatment affected gastric mucosal levels of TGF $\alpha$ and HB-EGF, both members of the epidermal growth factor receptor ligand family, and COX-2. The APE treatment did not affect TGF $\alpha$, HB-EGF and COX-2 expression; this rules out the hypothesis that these factors might be involved in APE gastro-protection. Aspirin-induced injury was associated with an increase in the expression of HB-EGF and COX-2 (both mRNA and protein), whereas TGF $\alpha$ expression was not affected by aspirin administration, thus suggesting that COX-2 and HB-EGF may play a role in the reparative events following aspirin injury. This is consistent with studies showing that oxidative stress increases gene expression of HB-EGF in cultured rat gastric epithelial cells ${ }^{(29)}$. Also, aspirin has been shown to rapidly up-regulate COX-2 mRNA expression in rat gastric mucosa, probably as a compensatory response to inhibition of COX-2 activity and gastrin PG synthesis ${ }^{(30,31)}$. COX-2 products play a relevant role for the maintenance of gastric mucosal integrity by preventing exogenous injury to the stomach and accelerating gastric mucosal healing ${ }^{(31,32)}$. TGF $\alpha$ mRNA expression has previously been shown to increase following treatment with acidified aspirin in the $\mathrm{rat}^{(33)}$. The apparent discrepancy between this and the present study might be accounted for by differences in the experimental design or methods to determine TGF $\alpha$ mRNA levels. However, the finding that HB-EGF, but not TGF $\alpha$, expression increased following aspirin treatment is not surprising. In fact, we previously demonstrated that exposure of gastric epithelial cells to Helicobacter pylori broth culture filtrates leads to an increased mRNA expression of HB-EGF but not of $\mathrm{TGF} \alpha^{(16)}$. Interestingly, APE pre-treatment counteracted the increased expression of HB-EGF and COX-2 induced by aspirin treatment. We postulate that the lower degree of gastric mucosa injury induced by aspirin in APE-pre-treated rats leads to a decreased need for the gastric mucosa to produce endogenous factors which promote mucosal healing. 
Considering that in the chronic aspirin study, rats were consuming approximately $10 \mathrm{ml} \mathrm{APE} \mathrm{at} 10^{-4} \mathrm{M} / \mathrm{d}$, the amount of phenolics consumed each day by each rat was about $0.9 \mathrm{mg} / \mathrm{kg}$. This is comparable with the amount of phenolic compounds consumed by an individual of $70 \mathrm{~kg}$ in weight eating two 'Annurca' apples per d (about $1 \mathrm{mg} / \mathrm{kg}$ ).

In conclusion, the present study shows that: (1) APE, a natural food extract obtained from a variety of apple (i.e. 'Annurca' apple), is able to prevent aspirin-induced injury (both acute and chronic) to the rat gastric mucosa through a mechanism independent of inhibition of gastric acid secretion; (2) this protective effect is at least in part contributed to by the antioxidant activity of APE; (3) aspirin causes up-regulation of HB-EGF and COX-2 expression in the gastric mucosa; (4) APE significantly counteracts aspirin-induced up-regulation of HB-EGF and COX-2 expression, probably as a consequence of the reduction in the extent of aspirin-induced injury. The present study gives further support to the concept that foodderived natural products may exert potential beneficial effects in the GI tract ${ }^{(34)}$. In particular, APE might be regarded as a safe and effective agent in the prevention of aspirin or other NSAID injury to the stomach. However, one must keep in mind that just because isolated compounds start from a natural food, they are not necessarily safe and natural. Future clinical studies are necessary to determine whether these compounds will be as interesting as preventive or therapeutic agents as they are in this pre-clinical study.

\section{Acknowledgements}

The study has been supported by the University research fund and therefore all authors have no conflict of interest to declare.

G. D. and M. R. designed the study and evaluated the results; G. M. and C. T. did the laboratory work; I. G. and A. G. G. did the data analysis and animal care; G. G. and V. F. did the APE preparation; all authors contributed to the drafting of the paper.

\section{References}

1. Bombardier C, Laine L, Reicin A, et al. (2000) Comparison of upper gastrointestinal toxicity of rofecoxib and naproxen in patients with rheumatoid arthritis. N Engl J Med 343, 1520-1528.

2. Niv Y, Battler A, Abuksis G, Gal E, Sapoznikov B \& Vilkin A (2005) Endoscopy in asymptomatic minidose aspirin consumers. Dig Dis Sci 50, 78-80.

3. Derry S \& Loke YK (2000) Risk of gastrointestinal haemorrhage with long-term use of aspirin: meta-analysis. BMJ 321, 1183-1187.

4. Chan FK, Ching JY, Hung LC, et al. (2005) Clopidogrel versus aspirin and esomeprazole to prevent recurrent ulcer bleeding. $N$ Engl J Med 352, 238-244.

5. Laheij RJ, Sturkenboom MC, Hassing RJ, Dieleman J, Stricker BH \& Jansen JB (2004) Risk of community-acquired pneumonia and use of gastric acid-suppressive drugs. JAMA 292, 1955-1960.

6. Yang YX, Lewis JD, Epstein S \& Metz DC (2006) Long-term proton pump inhibitor therapy and risk of hip fracture. JAMA 296, 2947-2953.

7. Garcia Rodriguez LA, Lagergren J \& Lindblad M (2006) Gastric acid suppression and risk of oesophageal and gastric adenocarcinoma: a nested case control study in the UK. Gut 55, 1538-1544.
8. Graziani G, D’Argenio G, Tuccillo C, Loguercio C, Ritieni A, Morisco F, Del Vecchio Blanco C, Fogliano V \& Romano M (2005) Apple polyphenol extracts prevent damage to human gastric epithelial cells in vitro and to rat gastric mucosa in vivo. Gut 54, 193-200.

9. Lommen A, Godejohann M, Venema D, Hollman PC \& Spraul M (2000) Application of directly coupled HPLC-NMR-MS to the identification and confirmation of quercetin glycosides and phloretin glycosides in apple peel. Anal Chem 72, 1793-1797.

10. Chomczynski P \& Sacchi N (1987) Single-step method of RNA isolation by acid guanidium thiocyanate-phenol-chloroform extraction. Anal Biochem 162, 156-159.

11. Tuccillo C, Manzo BA, D’Argenio G, et al. (2002) Up-regulation of heparin binding epidermal growth factor-like growth factor and amphiregulin expression in Helicobacter pylori-infected human gastric mucosa. Dig Liver Dis 34, 498-505.

12. Zarrilli R, Tuccillo C, Santangelo M, Nardone G \& Romano M (1999) Increased COX-2, but not COX-1, mRNA expression in Helicobacter pylori gastritis. Am J Gastroenterol 94, 3376-3378.

13. Mateos R, Lecumberri E, Ramos S, Goya L \& Bravo L (2005) Determination of malondialdehyde (MDA) by high-performance liquid chromatography in serum and liver as a biomarker for oxidative stress. Application to a rat model for hypercholesterolemia and evaluation of the effect of diets rich in phenolic antioxidants from fruits. J Chromatogr B 827, 76-82.

14. Mateos R, Goya L \& Bravo L (2004) Determination of malondialdehyde by liquid chromatography as the 2,4-dinitrophenylhydrazone derivative: a marker for oxidative stress in cell cultures of human hepatoma HepG2. J Chromatogr B Analyt Technol Biomed Life Sci 805, 33-39.

15. Guglietta A, Lesch CA, Romano M, McClure RW \& Coffey RJ (1994) Effect of transforming growth factor- $\alpha$ on gastric acid secretion in rats and monkeys. Dig Dis Sci 39, 177-182.

16. Romano M, Polk WH, Awad JA, Arteaga CL, Nanney LB, Wargovich MJ, Kraus ER, Boland CR \& Coffey RJ (1992) Transforming growth factor $\alpha$ protection against drug-induced injury to the rat gastric mucosa in vivo. J Clin Invest 90, 2409-2421.

17. Feng Y \& Besner GE (2007) Heparin-binding epidermal growth factor-like growth factor promotes enterocyte migration and proliferation in neonatal rats with necrotizing enterocolitis. J Pediatr Surg 42, 214-220.

18. Brzozowski T, Konturek PC, Konturek SJ, Pajdo R, Schuppan D, Drozdowicz D, Ptak A, Pawlik M, Nakamura T \& Hahn EG (2000) Involvement of cyclooxygenase (COX)-2 products in acceleration of ulcer healing by gastrin and hepatocyte growth factor. J Physiol Pharmacol 51, 751-773.

19. Cooke AR (1973) The role of acid in the pathogenesis of aspirin-induced gastrointestinal erosions and hemorrhage. Am J Dig Dis 18, 225-237.

20. Hunt RH \& Bazzoli F (2004) Should NSAID/low dose aspirin takers be tested routinely for $H$. pylori infection and treated if positive? Implications for primary risk of ulcer and ulcer relapse after initial healing. Alimet Pharmacol Ther 19, 9-16.

21. Ivey KJ (1988) Mechanism of nonsteroidal anti-inflamatory drug-induced gastric damage. Actions of therapeutic agents. Am J Med 84, Suppl. 2A, 41-48.

22. Singh P, Bhargava VK \& Garg SK (2002) Effect of melatonin and $\beta$-carotene on indomethacin induced gastric mucosal injury. Indian J Physiol Pharmacol 46, 229-234.

23. Alarcon de la Lastra C, Barranco MD, Martin MJ, Herrerias J \& Motiva V (2002) Extra-virgin olive oil-enriched diets reduce indomethacin-induced gastric oxidative damage in rats. $\mathrm{Dig}$ Dis Sci 47, 2783-2790.

24. Konturek SJ, Kitler ME, Brzozowski T \& Radecki T (1986) Gastric protection by meciadanol. A new synthetic flavonoid inhibiting histidine decarboxylase. Dig Dis Sci 31, 847-853. 
25. Mashita Y, Taniguchi M, Yokota A, Tanaka A \& Takeuchi K (2006) Oral but not parenteral aspirin upregulates COX-2 expression in rat stomachs. a relationship between COX-2 expression and PG deficiency. Digestion 73, 124-132.

26. Suzuki Y, Ishihara M, Segami T \& Ito M (1998) Anti-ulcer effects of antioxidants, quercetin, $\alpha$-tocopherol, nifedipine and tetracycline in rats. Jpn J Pharmacol 78, 435-441.

27. Rice-Evans CA, Miller NJ, Bolwell PG, Bramley PM \& Pridham JB (1995) The relative antioxidant activities of plantderived polyphenolic flavonoids. Free Radic Res 22, 375-383.

28. Peri L, Pietraforte D, Scorza G, Napolitano A, Fogliano V \& Minetti M (2005) Apples increase nitric oxide production by human saliva at the acidic $\mathrm{pH}$ of the stomach. A new biological function for polyphenols with a catechol group? Free Radic Biol Med 39, 668-681.

29. Miyazaki Y, Shinomura Y, Tsutsui S, Yasunaga Y, Zushi S, Higashiyama S, Taniguchi N \& Matsuzawa Y (1996) Oxidative stress increases gene expression of heparin-binding EGF-like growth factor and amphiregulin in cultured rat gastric epithelial cells. Biochem Biophys Res Commun 13, 542-546.
30. Davies NM, Sharkey KA, Asfaha S, Macnaughton WK \& Wallace JL (1997) Aspirin causes rapid up-regulation of cyclooxygenase- 2 expression in the stomach of the rats. Aliment Pharmacol Ther 11, 1101-1108.

31. Hatazawa R, Tanaka A, Tanigami M, Amagase K, Kato S, Ashida Y \& Takeuchi K (2007) Cyclooxygenase-2/prostaglandin E2 accelerates the healing of gastric ulcers via EP4 receptors. Am J Physiol 293, G788-G797.

32. Brzozowski T, Konturek PC, Sliwowski Z, Kwiecen S, Drozdowicz D, Pawlik M, Mach K, Konturek SJ \& Pawlik WW (2006) Interaction of nonsteroidal anti-inflammatory drugs (NSAID) with Helicobacter pylori in the stomach of humans and experimental animals. J Physiol Pharmacol 57, Suppl. 3, 67-79.

33. Polk WH, Dempsey PJ, Russell WE, Brown PI, Beauchamp RD, Barnard JA \& Coffey RJ Jr (1992) Redistribution of transforming growth factor $\alpha$ in rat gastric mucosa following acute injury. Gastroenterology 102, 1467-1474.

34. Ghosh S \& Playforf RJ (2003) Bioactive natural compounds for the treatment of gastrointestinal disorders. Clin Sci 104, $547-556$. 